九州大学学術情報リポジトリ

Kyushu University Institutional Repository

\title{
On a Multi-Level Sampling Inspection Plan for Cont inuous Production
}

Mikami, Misao

Kyushu University

https://doi.org/10.5109/12972

出版情報: 統計数理研究. 7 (1/2)，pp.1-10，1956-12. Research Association of Statistical Sciences

バージョン :

権利関係 : 


\title{
ON A MULTI-LEVEL SAMPLING INSPECTION PLAN FOR CONTINUOUS PRODUCTION
}

\author{
By \\ Misao Mrkami
}

(Received Sept. 19, 1956)

$\S 1$. Introduction. The sampling inspection plan for continuous production was first designed in 1943 by H. F. DODGE [1]." His plan, applicable only to characteristics subject to non-destructive inspection, is intended to have assurance that the percentage of defective units in accepted product will be held down to some prescribed low figure and so the Average Outgoing Quality (AOQ) function and Average Outgoing Quality Limit (AOQL) were derived. Afterwards, A. WALid and J. Wolfowirz [2] in 1945 and G. J. Lieberman [3] in 1953 discussed sampling inspection plans for continuous production which insure a prescribed limit on the outgoing quality even when production is not in statistical control. In 1955, H. F. DODGE [4] again designed a plan applicable not only to non-destructive inspection but also to destructive one. On the other hand, G. J. Lieberman and H. SoloMON [5] pointed out the inconvenient feature of plans, that is, the abrupt change between partial inspection and $100 \%$ inspection and introduced a multi-level sampling inspection plan applicable only to non-destructive inspection, which specifically allows for any number of sampling levels subject to the provision that transitions can only occur between adjacent levels.

In this paper, standing on the basis of the idea of G. J. LIEBERMAN and H. Solomon and generalizing their plan, the author wishes to propose a new multi-level sampling inspection plan in which we do not insist on $100 \%$ inspection even at the outset but always inspect a randomly chosen sample from every sequence composed of $N$ succssive individual units in continuous production line and which is applicable not only to non-destructive inspection but also to destructive one.

\section{§2. Procedures. The plan proposed in this paper is as follows:}

The infinite sequence of successive individual units produced by an essentially continuing process is to be devided into a group of infinitely many successive subsequences each of which consists of $N$ successive individual units in order of production and may be regarded as if a lot of size $N$ in an ordinary sense. For each subsequence of length $N$ (lot of size $N$ )

1) The number in bracket refers to the list of papers at the end. 
select a random sample of size $n_{j}$ at a sampling rate $f_{j}\left(n_{j}=N f_{j}\right)$ and decide whether the lot of $N$ in consideration is acceptable or not for acceptance number $c=0$. In other words, if no defects are detected in the sample of $n_{j}$, the lot of $N$ is to be accepted and if one or more defects are detected, then the lot is to be rejected. For convenience' sake, the sampling at rate $f_{j}$ will be called $\left(f_{j}\right)$-sampling or more simply $\left(f_{j}\right)$. Then,

$0)$ Starting with the $\left(f_{0}\right)$-sampling inspection, continue such inspection until $i_{0}$ samples in succession are found clear of defects;

1) When, at rate $f_{0}, i_{v}$ samples in succession are found clear of defects, discontinue $\left(f_{w}\right)$-sampling and turn to $\left(f_{1}\right)$-sampling. If the next $i_{1}$ inspected samples in succession are non-defective, proceed to the next level $\left(f_{2}\right)$; if a defective occurs, revert immediately to $\left(f_{0}\right)$-sampling;

2) When at rate $f_{2}, i_{2}$ inspected samples in succession are found clear of defects, discontinue $\left(f_{2}\right)$-sampling and proceed to the next level $\left(f_{3}\right)$; if a defective occurs, revert immediately to $\left(f_{1}\right)$-sampling;

h) When at rate $f_{n}, i_{n}$ inspected samples in succession are found clear of defects, discontinue $\left(f_{n}\right)$-sampling and proceed to the next level $\left(f_{h+1}\right)$; if a defective occurs, revert immediately to $\left(f_{h-1}\right)$-sampling;

$k-1)$ When at rate $f_{k-1}, i_{k-1}$ inspected samples in succession are found clear of defects, discontinue $\left(f_{k-1}\right)$-sampling and proceed to the next level $\left(f_{k}\right)$; if a defective occurs revert immediately to $\left(f_{k-2}\right)$-sampling;

k) When in operation of $\left(f_{k}\right)$-sampling, a defective occurs in a sample, revert immediately to $\left(f_{k-1}\right)$-sampling, otherwise, continue $\left(f_{k}\right)$-sampling.

Though, generally speaking, all the $f_{j}$ 's can take any values in the interval $(0,1)$ including 1 and $k$ can run over any integer from 0 to $\infty$, it is naturally assumed for practical use that $1 \geqq f_{0}>f_{1}>f_{2}>\cdots>f_{k}>0$ and $k$ may of ten be a relatively small integer in circumstances.

This plan will be called the multi-level continuous sampling inspection plan following to the denomination of LIEBERMAN and SoLOMON. But our plan is to be distinguished from their plan in the point that, in the latter, selected samples are always regarded as of size 1, while in the former the sizes of samples are generally greater than one and therefore, by adopting the acceptance number $c=0$ our plan becomes practically applicable both to destructive inspection and to non-destructive one.

§3. Study of the procedure as a Markov chain. It is useful for theoretical discussion to relate our procedure to a Markov chain. Let the process of production is of average fraction defective $p$. Then the probability $p_{j}$, that a lot of $N$ subject to $\left(f_{j}\right)$-sampling inspection is accepted with respect to the acceptance number $c=0$, is given by 


$$
\begin{aligned}
p_{j} & =\left(\begin{array}{c}
N-N p \\
n_{j}
\end{array}\right)\left(\begin{array}{l}
N \\
n_{j}
\end{array}\right)=\frac{(N-N p)(N-N p-1) \cdots\left(N-N p-n_{j}+1\right)}{N(N-1) \cdots\left(N-n_{j}+1\right)} \\
& =(1-p)\left(1-\frac{N}{N-1} p\right) \cdots\left(1-\frac{N}{N-}-\frac{N}{n_{j}+1} p\right) .
\end{aligned}
$$

Since the relation

$$
(1-p)^{n_{j}}>p_{j}>\left(1-\frac{N}{N-n_{j}+1} p\right)^{n_{j}}
$$

holds good, if $n_{j}$ is sufficiently small compared with $N$, then $p_{j}$ can be approximated by $(1-p)^{n}$; moreover, if $p$ is also sufficiently small, $p_{j}$ by $e^{-p n_{j}}$.

Consider a sequence of sampling inspections. At anytime $m$ (or after the $m$-th sampling inspection) the system is in one of the states

$$
E_{v}\left(v=0,1,2, \cdots, i_{0}, \cdots, i_{0}+i_{1}, \cdots, i_{0}+i_{1}+i_{2}, \cdots, i_{0}+i_{1}+\cdots+i_{k-1}+1\right)
$$

where

$E_{i_{1}+i_{1}+\cdots+i_{j}+h}\left(0 \leqq j \leqq k-2,0 \leqq h \leqq i_{i+1}-1\right)=$ state where the $(m-h)$-th trial resulted in beginning $\left(f_{j}\right)$-sampling and the last $h$ samples were free of defective,

or, in full details,

$E_{0}=$ state where the $m$-th trial resulted in beginning $\left(f_{10}\right)$-sampling; this means that, in procession of $\left(f_{11}\right)$ - or $\left(f_{1}\right)$-sampling, one or more defectives was detected in the $m$-th sample;

$E_{1}=$ state where the $(m-1)$-th trial resulted in beginning $\left(f_{0}\right)$-sampling and the $m$-th sample was free of defective;

$E_{2}=$ state where the $(m-2)$-th trial resulted in beginning $\left(f_{1}\right)$-sampling and where the $(m-1)$-th and $m$-th trials were free of defective;

$E_{i_{0-l}}=$ state where the $\left(m-i_{0}+1\right)$-th trial resulted in beginning $\left(f_{0}\right)$ sampling and the last $\left(i_{0}-1\right)$ samples were free of defective;

$E_{t_{0}}=$ state where the $m$-th trial resulted in beginning $\left(f_{1}\right)$-sampling: this means that the $\left(m-i_{v}\right)$-th trial had resulted in beginning $\left(f_{0}\right)$-sampling and the last $i_{0}$ samples were free of defective, or, in procession of $\left(f_{2}\right)$ sampling, one or more defectives were detected in the $m$-th sample;

$E_{l_{10}+1}=$ state where the $(m-1)$-th trial resulted in beginning $\left(f_{1}\right)$ sampling and the $m$-th sample was free of defective;

$E_{l_{\mathrm{u}+i_{1}-1}}=$ state where the $\left(m-i_{1}+1\right)$-th trial resulted in beginning $\left(f_{1}\right)-$ sampling and the last $i_{1}-1$ samples were free of defective;

$E_{l_{1++41}}=$ state where the $m$-th trial resulted in beginning $\left(f_{2}\right)$-sampling: this means that the $\left(m-i_{1}\right)$-th trial had resulted in beginning $\left(f_{1}\right)$-sampling and the next $i_{1}$ samples in succession were free of defective, or in procession of $\left(f_{3}\right)$-sampling one or more defectives were detected in the $m$-th sample; 
$E_{\mathrm{t}_{1}+i_{1}+1}=$ state where the $(m-1)$-th trial resulted in beginning $\left(f_{2}\right)$ sampling and the $m$-th sample was free of defective;

$E_{l_{0}+i_{1}+t_{2}}=$ state where the $\boldsymbol{m}$-th trial resulted in beginning $\left(f_{3}\right)$-sampling;

$E_{i_{1}+i_{1}+\cdots+i_{k-1}-1}=$ state where the $\left(m-i_{k-1}+1\right)$-th trial resulted in beginning $\left(f_{k-1}\right)$-sampling and the last $i_{k-1}-1$ samples were free of defective; and

$E_{l_{0}+i_{1}+\cdots+i_{k-1}}=$ state where the $m$-th trial resulted in beginning $\left(f_{k}\right)-$ sampling ;

$E_{t_{1}+i_{1}+\cdots+t_{k-1}+1}=$ state where the $(m-1)$-th trial resulted in beginning $\left(f_{k}\right)$-sampling or state where in procession of $\left(f_{k}\right)$-sampling no defectives were detected in the $m$-th sample.

Then the transition probability $p_{r, s}$ from a state $E_{r}$ to a state $E_{s}(r$, $\left.s=0,1,2, \cdots, i_{0}+i_{1}+i_{2}+\cdots+i_{k-1}+1\right)$ is given by following;

$$
\begin{array}{r}
p_{i_{0}+i_{1}+\cdot+i_{j}-h, i_{0}+i_{1}+\cdots+i_{j}-h+1}=p_{j}, \quad p_{i_{0}+i_{1}+\cdots+i_{j}-h, h_{1}+\cdots+i_{j-2}}=q_{j} \\
\text { for } 0 \leqq j \leqq k-1, \quad 1 \leqq h \leqq i_{j}
\end{array}
$$

or, in full details,

$$
\begin{aligned}
& p_{r, 0}=q_{i n}, \quad p_{r . r+1}=p_{0} \quad \text { for } r=0,1, \cdots, i_{0}-1 \text {; } \\
& p_{r, 0}=q_{1}, \quad p_{r, r+1}=p_{1} \quad \text { for } r=i_{0}, i_{0}+1, \cdots, i_{0}+i_{1}-1 \text {; } \\
& p_{r, i_{0}}=q_{2}, \quad p_{r_{1} r+1}=p_{2} \quad \text { for } r=i_{v}+i_{1}, i_{0}+i_{1}+1, \cdots, i_{0}+i_{1}+i_{2}-1 \text {; } \\
& \text { (1, } \left.{ }^{\prime}\right) \quad p_{r, i_{0}+i_{1}}=q_{3}, \quad p_{r, r+1}=p_{3} \quad \text { for } r=i_{0}+i_{1}+i_{2}, \cdots, i_{0}+i_{1}+i_{2}+i_{3}-1 \text {; } \\
& p_{r, i_{1}+\cdot+i_{k-3}}=q_{k-1}, \quad p_{r, r+1}=p_{k-1} \\
& \text { for } r=i_{1}+\cdots+i_{k-2}, \cdots, i_{0}+\cdots+i_{k-1}-1
\end{aligned}
$$

and

$$
\begin{aligned}
& p_{r, i_{u}+\cdots+i_{k-2}}=q_{k}, p_{r, i_{0}+\cdots+i_{k-1}+1}=p_{k} \\
& \text { for } r=i_{0}+\cdots+i_{k-1}, i_{0}+\cdots+i_{k-1}+1 ;
\end{aligned}
$$

all other $p_{r_{, s}}=0$.

This type of Markov chain is aperiodic and irreducible, so that the probability of finding the system at time $m$ in state $E_{\mathrm{v}}$ converges to a unique determined limit which is independent of the initial distribution. Moreover, since it is a finite chain, it cannot contain any null-states and therefore no state is transient. Hence, we have recurrent states and the limits

$$
\lim _{n \rightarrow \infty} p_{v v}^{(n)}=u_{c}>0 \quad\left(v=0,1, \cdots, i_{0}+i_{1}+\cdots+i_{s-1}+1\right)
$$

exist, which are independent of $j$. The quantity $u_{v}$ is nothing but the reciprocal of mean recurrence time and satisfies the relations $\sum u_{h}=1$ and

$$
u_{v}=\sum_{h} u_{h} p_{v i n} \text {. }
$$


In a finite chain the recurrence time of any state $E_{\mathrm{o}}$ has a finite variance and the weak law of large numbers holds good for the number of passages through $E_{0}$. Thus, when $g_{j}^{*(n)}$ is the number of samples in a state $E_{\mathrm{v}}$ in the first $n$ trials, $E\left[g_{3}^{*(n)}\right]=u_{\mathrm{v}}$ is finite and we get

$$
\lim _{n \rightarrow \infty} P\left(\left|\frac{g_{1}^{*(n)}}{n}-u_{v}\right|>\varepsilon\right)=0 \text {. }
$$

Now the number of times $g_{j}^{(n)}$ that $\left(f_{j}\right)$-sampling is applied in the first $n$ trials is a finite sum of $g_{j}^{*(n)}$ for $E_{v}$ and the probability $P_{j}$ that for a randomly chosen $m, x_{m}$ is the result of $\left(f_{j}\right)$-sampling is also a finite sum of $u_{v}$ of the same form as that of $g_{j}^{*(n)}$, we find

$$
\lim _{n \rightarrow \infty} P\left(\left|\frac{g_{i}^{(n)}}{n}-P_{3}\right|>\varepsilon\right)=0 .
$$

Now, let $f_{j_{m}}$ be the sampling ratio attacted to $x_{m}$ in the sequence $\left(x_{1}\right.$, $\left.x_{2}, \cdots, x_{m}, \cdots\right)$, then the average number of inspected individual units is given by

$$
\lim _{n \rightarrow \infty} \frac{1}{n} \sum_{m=1}^{n} N f_{j_{m}},
$$

provided the limit exists. Furthermore, the expression (6) can be rewritten as

$$
\lim _{n \rightarrow \infty} \frac{N}{n} \sum_{j=0}^{k} f_{j} g_{j}^{(n)} .
$$

Thus, in virtue of (5), it is easily found that (6) is equivalent to the expression

$$
F=\sum_{j=v}^{k} N f_{j} P_{j} .
$$

Before ending this paragraph, let us see that it is not difficult to find the values of $u_{\mathrm{r}}$ 's.

The system of equations ( 3$)$ can be written down in aid of $\left(1_{1}^{\prime}\right)$ and $\left(1_{2}\right)$ as follows:

$$
\begin{aligned}
& \boldsymbol{u}_{0}=q_{0} \sum_{r=0}^{i_{0}-1} u_{r}+q_{1} \sum_{r=t_{0}}^{i_{0}+t_{0}-1} u_{r}, \\
& u_{u_{0}}=p_{0} u_{t_{0}-1}+q_{2} \sum_{r=i_{0}+i_{1}}^{i_{0}+t_{1}+i_{2}-1} u_{r} \text {, } \\
& u_{i_{u}+i_{1}}=p_{1} u_{i_{1}+i_{1}-1}+q_{3} \sum_{r=i_{0}+i_{1}+i_{2}}^{i_{0}+i_{1}+i_{2}+i_{3}-1} u_{r} \text {, } \\
& u_{i_{0}+i_{1}+\cdots+i_{k-3}}=p_{k-3} u_{i_{0}+i_{1}+\cdots+i_{k-3}-1}+q_{k-1} \sum_{r=i_{1}+i_{1}+\cdots+i_{k}+2}^{i_{0}+\cdots+i_{k-1}-1} u_{r}
\end{aligned}
$$




$$
\mid \begin{aligned}
u_{i_{0}+i_{1}+\cdots+i_{k-2}} & =p_{k-2} u_{i_{0}+\cdots+i_{k-2}-1}+q_{k} \sum_{r=i_{0}+\cdots+i_{k-1}}^{i_{0}+\cdots+i_{k-1}+1} u_{r}, \\
u_{t_{0}+\cdots+i_{k-1}} & =p_{k-1} u_{i_{0}+\cdots+i_{k-1}-1}, \\
u_{i_{0}+\cdots+i_{k-1}+1} & =p_{k} u_{i_{1}+\cdots+i_{k-1}}+p_{k} u_{i_{1}+\cdots+i_{k-1}+1}, \\
u_{s} & =p_{0} u_{s-1} \quad\left(1 \leqq s \leqq i_{0}-1\right), \\
u_{i_{1}+i_{1}+\cdots+i_{j+l}} & =p_{j} u_{i_{0}+\cdots+i_{j}+t-1} \quad\left(0 \leqq j \leqq k-1, \quad 1 \leqq t \leqq i_{j+1}-1\right),
\end{aligned}
$$

whence we have

$$
\begin{aligned}
u_{s} & =p_{v}^{s} u_{v}, \\
u_{i_{0}+i_{1}+\cdots+i_{j}} & =p_{j}^{t_{j}} u_{i_{0}+\cdots+i_{j-1}}+\left(1-p_{j+2}^{i j+2}\right) u_{w_{0}+\cdots+i_{j+1}} \quad(0 \leqq j \leqq k-3) . \\
u_{i_{0}+i_{1}+\cdots+i_{k-2}}=p_{k-2}^{i_{k-2}} u_{i_{0}+\cdots+i_{k-3}}+q_{k}\left(p_{k-1} u_{i_{u}+\cdots+i_{k-1}-1}+p_{k} u_{i_{0}+\cdots+i_{k-1}}\right. & \left.+p_{k} u_{i_{0}+\cdots+i_{k-1}+1}\right)
\end{aligned}
$$

and therefore

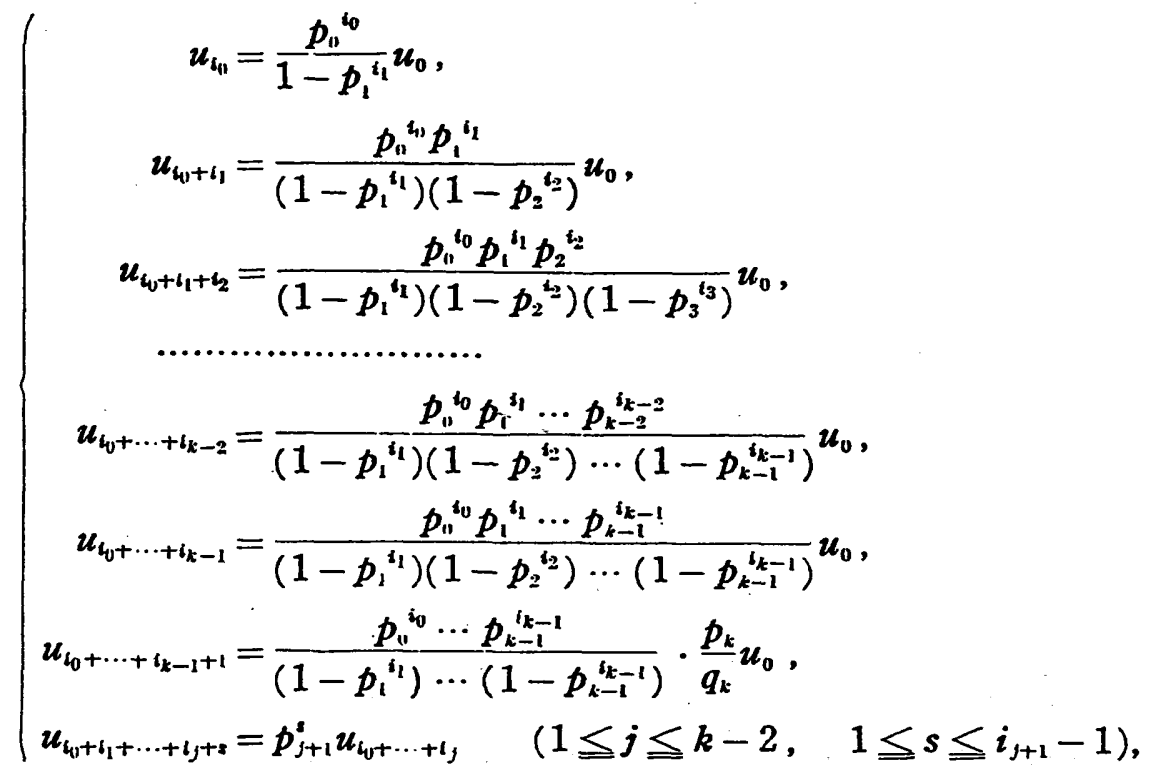

where the first part of (9) can be summarized in the form

$$
u_{i_{0}+i_{1}+\cdots+i_{3}}=\left(\prod_{n=0}^{j} p_{A}^{i_{n}}\right) u_{0} / \prod_{n=1}^{j+1}\left(1-p_{k}^{i_{h}}\right) \quad(0 \leqq j \leqq k-2) .
$$

Now, substituting the expression for $u_{v}\left(v=0,1,2, \cdots, i_{0}+i_{1}+\cdots+i_{k-1}+1\right)$ given by ( 9 ) into the relation

$$
\sum_{v=0}^{i_{v}+\cdots+i_{k-1}+1} u_{v}=1
$$

we obtain a linear equation for only one unknown $u_{0}$ which is of course easily soluble and thereafter we can find the expressions for all the $u_{v}$ 's of the arguments $p,(0 \leqq s \leqq k)$ in aid of (9). 
§4. Calculations of $\boldsymbol{P}_{j}^{\prime}$ s. We have already defined the probability $P_{j}$ that a sample is sampled out by operation of $\left(f_{j}\right)$-sampling. Moreover, for convenience' sake, let us define the probability $P_{j}^{\prime}$ that a level $(j)$ or $\left(f_{j}\right)$-sampling begins at time $m$. In other words, $P_{j}^{\prime}$ represents the probability that for randomly chosen $m, x_{m}$ is the result of the $m$-th sampling inspection with $\left(f_{j}\right)$-sampling and $x_{m-t_{j-1}}=0, \cdots, x_{m-1}=0$ while $\left(f_{j-1}\right)$-sampling or $x_{m-1}=1$ while $\left(f_{j+1}\right)$-sampling in operation. Especially, $P_{0}^{\prime}$ is the probability that for a randomly chosen $m, x_{m}$ is the result of the $\left(f_{11}\right)$ sampling and $x_{m-1}=1$ while $\left(f_{10}\right)$-sampling or $\left(f_{1}\right)$-sampling. And, $P_{k}^{\prime}$ is the probability that for a randomly chosen $m, x_{m}$ is the result of $\left(f_{k}\right)$-sampling and $x_{m-i_{k-1}}=0, \cdots, x_{m-1}=0$ while $\left(f_{k-1}\right)$-sampling in operation.

Then we have

$$
\begin{aligned}
P_{0}^{\prime} & =P_{0}^{\prime}\left(q_{0}+p_{0} q_{0}+p_{10}^{2} q_{0}+\cdots+p_{0}^{i_{11}-1} q_{0}\right) \\
& +P_{1}^{\prime}\left(q_{1}+p_{1} q_{1}+p_{1}^{2} q_{1}+\cdots+p_{1}^{i_{1}-1} q_{1}\right) \\
& =P_{0}^{\prime}\left(1-p_{0}^{i_{0}}\right)+P_{1}^{\prime}\left(1-p_{1}^{\prime \prime}\right)
\end{aligned}
$$

and similarly

$$
\begin{aligned}
& P_{1}^{\prime}=P_{0}^{\prime} p_{0}^{b_{1}}+P_{2}^{\prime}\left(q_{2}+p_{2} q_{2}+\cdots+p_{2}^{t_{2}-1} q_{2}\right) \\
& =P_{0}^{\prime} p_{0}^{k_{0}}+P_{1}^{\prime}\left(1-p_{1}^{i_{1}}\right) \text {, } \\
& P_{:}^{\prime}=P_{1}^{\prime} p_{1}^{{ }^{i_{1}}}+P_{3}^{\prime}\left(1-p_{3}^{{ }^{{ }^{3}}}\right) \text {, } \\
& P_{k-2}^{\prime}=P_{k-3}^{\prime} p_{k-3}^{i_{k-3}}+P_{k-1}^{\prime}\left(1-p_{k-1}^{i_{k-1}}\right), \\
& P_{k-1}^{\prime}=P_{k-2}^{\prime} p_{k-2}^{i_{k}-2}+P_{k}{ }^{\prime}\left(q_{k}+p_{k} q_{k}+p_{k}{ }^{2} q_{k}+\cdots \text { ad inf }\right) \\
& =P_{k-2}^{\prime} p_{k-2}^{i_{k-2}}+P_{k}^{\prime}, \\
& P_{k}^{\prime}=P_{k-1}^{\prime} p_{k-1}^{{ }^{i_{k-1}}} \text {. }
\end{aligned}
$$

However, it is easily found that

$$
\begin{aligned}
& P_{0}=P_{0}^{\prime}\left(1+p_{0}+p_{0}{ }^{2}+\cdots+p_{0}{ }^{i_{0}-1}\right)=P_{0}{ }^{\prime}\left(1-p_{0}{ }^{i_{11}}\right) / q_{0}, \\
& P_{1}=P_{1}^{\prime}\left(1+p_{1}+p_{1}{ }^{2}+\cdots+p_{1}{ }^{i_{1}-1}\right)=P_{1}{ }^{\prime}\left(1-p_{1}^{{ }^{1}}\right) / q_{1} \text {, } \\
& P_{2}=P_{2}^{\prime}\left(1-p_{2}{ }^{t_{2}}\right) / q_{2} \text {, } \\
& P_{k-2}=P_{k-2}^{\prime}\left(1-p_{k-2}^{i_{k-2}}\right) / q_{k-2}, \\
& P_{k-1}=P_{k-1}^{\prime}\left(1-p_{k-1}^{k_{k-1}}\right) / q_{k-1} \text {, } \\
& P_{k}=P_{k}^{\prime}\left(1+p_{k}+p_{k}{ }^{2}+\cdots \text { ad inf }\right)=P_{k}^{\prime} / q_{k}
\end{aligned}
$$

or

$$
\begin{aligned}
& P_{1}^{\prime}=P_{0}{ }^{\prime} p_{0}{ }^{i_{10}} /\left(1-p_{1}^{{ }^{l_{1}}}\right) \text {, } \\
& P_{2}^{\prime}=P_{0}^{\prime} p_{11}^{t_{0}} p_{1}^{i_{1}} /\left\{\left(1-p_{1}^{t_{t}}\right)\left(1-p_{2}^{t_{2}}\right)\right\} \text {, } \\
& P_{3}^{\prime}=P_{0}^{\prime} p_{0}^{i^{t_{0}}} p_{1}^{{ }^{t_{1}}} p_{2}^{i_{2}} /\left\{\left(1-p_{1}^{i_{1}}\right)\left(1-p_{2}{ }^{i_{2}}\right)\left(1-p_{3}{ }^{i_{3}}\right)\right\} \text {, }
\end{aligned}
$$




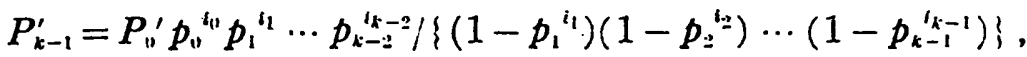

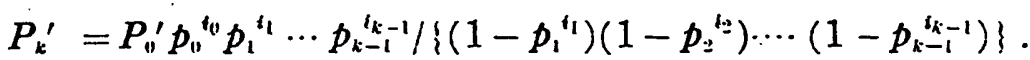

And therefore, we obtain

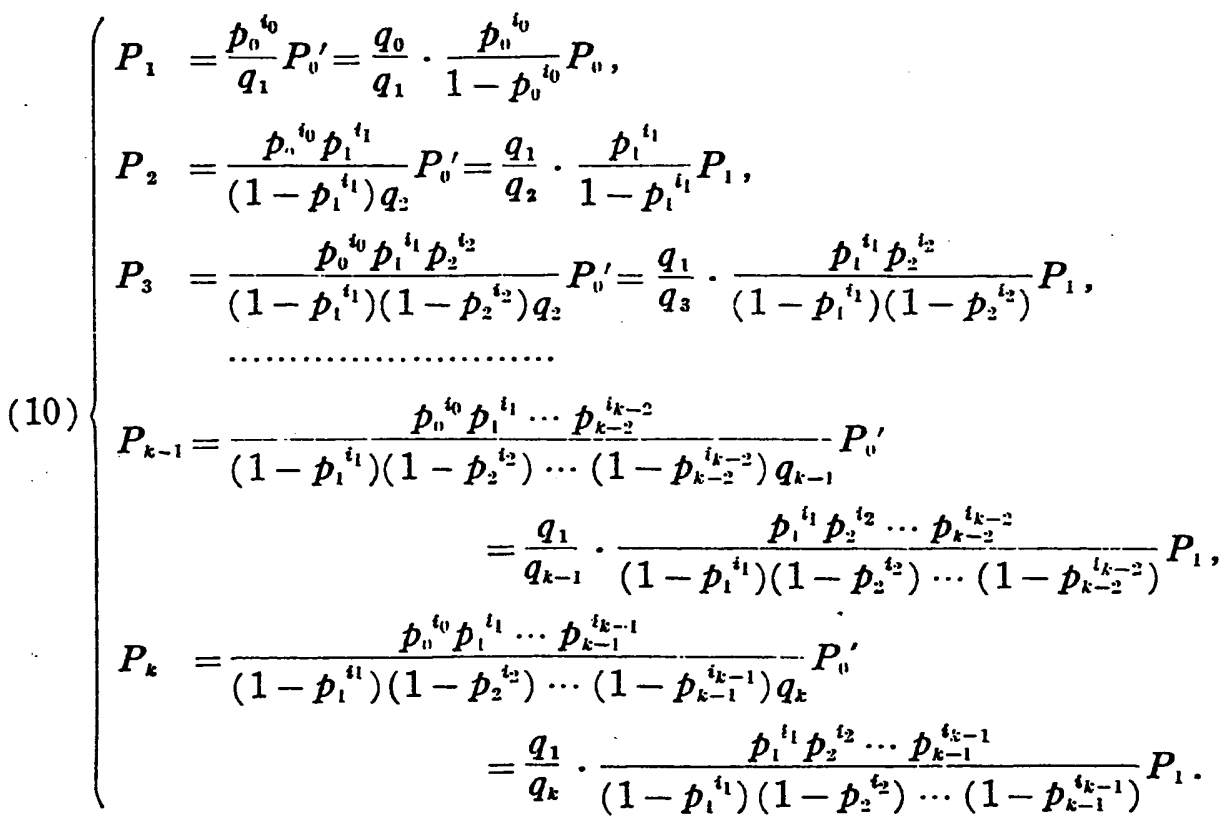

Substituting these expressions of $P_{j}^{\prime}$ s into the equation $\sum_{j=0}^{k} P_{j}=1$, we get

$$
\begin{aligned}
& q_{0} P_{0}\left\{\frac{1}{q_{0}}+\frac{1}{q_{1}} \cdot \frac{p_{11}{ }^{i_{0}}}{1-p_{11}{ }^{{ }_{11}}}+\frac{1}{q_{2}} \cdot \frac{p_{10}{ }^{{ }_{10}} p_{1}^{i_{1}}}{\left(1-p_{0}{ }^{i_{11}}\right)\left(1-p_{1}{ }^{i_{1}}\right)}+\cdots\right. \\
& \left.+\frac{1}{q_{k}} \cdot \frac{p_{0}^{i_{10}} \cdots p_{k-1}^{i_{k}-1}}{\left(1-p_{0}^{i_{i}}\right) \cdots\left(1-p_{k-1}^{i_{k}-1}\right)}\right\}=1
\end{aligned}
$$

from which $P_{0}$ can be uniquely determined provided that the set of values of $i$ 's and $f_{j}^{\prime} s$ is specified.

§5. Lot quality protection. It is evident that, if a set of values of the constants $f_{j}$ and $i_{j}(j=0,1,2, \cdots, k)$ which characterize the sampling inspection plan is suitably selected, the operating characteristic (OC) curve can be determined.

In this paragraph we shall show that a set of values of $f_{i}$ and $i_{j}$ can be determined on the standpoint of lot quality protection by which we can establish a scheme applicable to destructive inspection.

Since a lot of size $N$ is subject to $\left(f_{f}\right)$-sampling inspection with the probability $P_{j}$ and then is accepted with the conditional probability $p_{j}$, the expected value of the probability that a lot of size $N$ is accepted is given by 


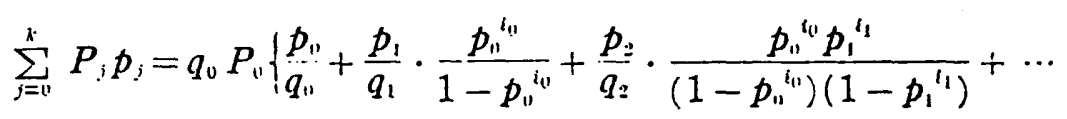

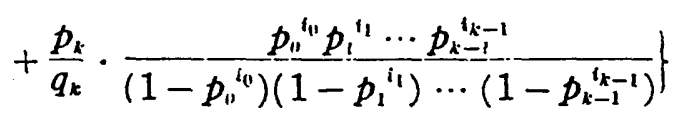

and the expected value of the probability that a lot of size $N$ is rejected by

$$
\sum_{j=0}^{k} P_{j} q_{j}=1-\sum_{j=1}^{k} P_{j} p_{j}
$$

Now, let the consumer's risk $\alpha$ and producer's risk $\beta$ be specified, then a suitable choice of fraction defectives $p^{\prime}$ and $p^{\prime \prime}$ determines the four points $(0,1),\left(p^{\prime \prime}, 1-\beta\right),\left(p^{\prime}, \alpha\right),(1,0)$ on the OC-curve. So that, we can determine the multi-level sampling inspection scheme by such set of $f_{j}$ 's (or $n_{j}$ 's) and $i$,'s that the system of simultaneous equations

$$
\begin{aligned}
& \sum_{j=1}^{k} P_{,} p_{j}=\alpha, \\
& \sum_{j=0}^{k} P_{j} q_{j}{ }^{\prime \prime}=\beta
\end{aligned}
$$

is satisfied, where $p_{j}^{\prime}$ or $q_{j}^{\prime \prime}\left(=1-p_{j}^{\prime \prime}\right)$ are corresponding values of $p_{j}$ or $q_{j}$ calculated for the case $p=p^{\prime}$ or $p=p^{\prime \prime}$ respectively.

As an example let us single out a special case $k=1$, then (15) reduces to

$$
q_{0} P_{0}\left\{1+p_{01}^{{ }^{k_{11}}} /\left(1-p_{0}{ }^{{ }^{\prime \prime}}\right)\right\}=\beta
$$

in which, for convenience' sake of printing, we temporarily omit prime to be attached to $q_{0}$ or $p_{0}$. In virtue of the relation $\Sigma P_{j}=1$, the equation (16) gives rise to

$$
\left(1-p_{0}^{i_{0}}\right) \beta\left(\frac{1}{q_{0}}+\frac{1}{q_{1}} \frac{p_{11}^{i_{0}}}{1-p_{11}^{i_{0}}}\right)=1
$$

or

$$
p_{v}^{t_{0}}\left(\frac{1}{q_{11}}-\frac{1}{q_{1}}\right)=\frac{1}{\beta}-\frac{1}{q_{11}}
$$

Thus, recovering prime to $p_{0}$ or $q_{0}$, we have

$$
i_{0} \log p_{0}{ }^{\prime \prime}==\log \frac{q_{1}^{\prime \prime}\left(q_{1}^{\prime \prime}-\beta\right)}{\beta\left(q_{1}^{\prime \prime}-q_{0}^{\prime \prime}\right)}
$$

and similarly from (13)

$$
i_{v} \log p_{0}^{\prime}=\log \frac{q_{1}{ }^{\prime}\left(q_{10}{ }^{\prime}-1+\alpha\right)}{(1-\alpha)\left(q_{\mathrm{t}}{ }^{\prime}-q_{11}{ }^{\prime}\right)} .
$$

Therefore, given the alues of $\alpha, \beta, p^{\prime}$ and $p^{\prime \prime}$, we obtain $\infty^{\prime}$ sets of values of $n_{0}, n_{1}$ and $i_{0}$ from (17) and (18). So that, by specifying a ratio of $n_{0}$ 
and $n_{1}$ or $f_{0}$ and $f_{1}$, we can uniquely determine a set of values of $n_{0}, n_{1}$ and $i_{v}$ and accordingly the sampling inspection plan.

Though it is desirable to determine our plan explicitly and tabulate the results for various values of $k$, we postpone them a little longer. And also another development of our idea on different standpoints of protection of interests of producer or consumer which may be considered more adaptable to practical use, will be expected to appear in the next paper.

\section{KYUSHU UNIVERSITY}

\section{Literature}

[1]. H. F. Dodar; A sampling inspection plan for continuous production. Ann. Math. Stat., 14 (1943), 264-279.

[2] A. WALD and J. WoLfowitz; Sampling inspection plans for continuous production which insure a prescribed limit on the outgoing quality. Ann. Math. Stat., 16 (1945), 30-49.

[3] G. J. Lieberman; $A$ note on Dodge's continuous inspection plan. Ann. Math. Stat., 24 (1953), 480-484.

[4] H. F. DODGE; Chain sampling inspection plan. Industrial Quality Control, 11 (1955), 10-13.

[5] G. J. Lieberman and H. Solomon; Multi-level continuous sampling plans. Ann. Math. Stat., 26 (1955), 686-704. 